\title{
Lymnaea Epidermal Growth Factor Promotes Axonal Regeneration in CNS Organ Culture
}

\author{
Willem C. Wildering, Petra M. Hermann, and Andrew G. M. Bulloch \\ Department of Physiology and Biophysics, Neuroscience Research Group, Faculty of Medicine, Health Sciences Center, \\ University of Calgary, Calgary, Alberta, Canada, T2N 4N1
}

\begin{abstract}
Members of the epidermal growth factor (EGF) family are frequently implicated in the injury response of the mammalian nervous system. Although this implication is supported by extensive molecular evidence, it is not underpinned by conclusive functional data. Recently, we found that expression of an EGF homolog from the pond snail Lymnaea stagnalis (L-EGF) is upregulated after axotomy in the adult CNS, suggesting a role for this molecule in the injury response of the CNS. In the present study we asked whether L-EGF can promote axonal regeneration of three types of identified neurons in organcultured CNS. Treatment with purified L-EGF substantially enhanced axonal regeneration of all three types of neurons, an effect inhibited by submicromolar doses of PD153035, a specific EGF receptor (EGFR) tyrosine kinase inhibitor. In addition, PD153035 and K252a, a nonspecific kinase inhibitor, also reduced the degree of axonal regeneration that occurs without
\end{abstract}

L-EGF supplementation, indicating that L-EGF or other EGFR ligands synthesized in the CNS participate in the regenerative response. An intriguing aspect of these results is that axonal regeneration of different, intrinsically L-EGF responsive and unresponsive neurons occurred in a coordinated manner. This observation suggests that indirect in addition to direct actions contribute to the beneficial effect of L-EGF. In conclusion, we provide functional evidence that an EGF homolog can promote axonal regeneration, substantiating existing molecular evidence implicating the EGF family in peripheral nerve regeneration and emphasizes the therapeutic potential of these molecules.

Key words: neurotrophic factor; epidermal growth factor; axonal regeneration; invertebrate; mollusk; CNS; L-EGF; peripheral nerve regeneration; neurotrauma
Traumatic injury to the mammalian nervous system triggers complex physiological responses from both injured neurons and glial populations and involves a variety of secreted molecules, including cytokines and various polypeptide growth factors (for review, see Logan et al., 1994; Ambron and Walters, 1996; Ide, 1996; Ebadi et al., 1997; Fu and Gordon, 1997; Frostick et al., 1998; Raivich et al., 1999; Streit et al., 1999; Terenghi, 1999; Goldberg and Barres, 2000). There is extensive evidence pointing to a role of the epidermal growth factor (EGF) family (e.g., EGF, heparin binding-EGF, TGF- $\alpha$, and neuregulins) in the injury response of the central and peripheral mammalian nervous (Toma et al., 1992; Xian and Zhou, 1999, 2000). Unfortunately, most studies on neurotrophic/neuroprotective actions of EGF homologs have been limited to in vitro assays (Morrison et al., 1988; Ferrair et al., 1991; Chalazonitis et al., 1992; Kimpinski and Mearow, 2001). Consequently, very little or no data are available on the physiological actions of members of this family in the CNS in vivo (Ferrair et al., 1991; Peng et al., 1998; Justicia and Planas, 1999)

Received April 25, 2001; revised July 24, 2001; accepted Sept. 11, 2001.

This work was supported by grants from the Canadian Institutes of Health Research and The Human Frontiers Science Program Organization (RG0045/ 1997B). W.C.W. was supported by the Alberta Heritage Foundation for Medical Research (AHFMR). A.G.M.B. is an AHFMR Scientist. We thank Dr. G. T. Nagle (University of Texas Medical Branch, Galveston, TX) for kindly providing purified L-EGF.

W.C.W. and P.M.H. contributed equally to this study.

Correspondence should be addressed to Dr. W. C. Wildering, Department of Physiology and Biophysics, Neuroscience Research Group, Faculty of Medicine, Health Sciences Center, University of Calgary, 3330 Hospital Drive N.W., Calgary, Alberta, Canada, T2N 4N1. E-mail: wilderin@ucalgary.ca.

Copyright (C) 2001 Society for Neuroscience $0270-6474 / 01 / 219345-10 \$ 15.00 / 0$ or on their actions during peripheral nerve regeneration (Dubuisson et al., 1993).

This paper investigates the effects of an endogenous EGF homolog on axonal regeneration in the gastropod mollusk Lymnaea stagnalis. This molecule (L-EGF) is a member of the EGF family, i.e., it shares the characteristic cysteine framework and acidic $\mathrm{C}$ terminal amino acid residues with other mammalian and invertebrate members of this family (Hermann et al., 2000b). Unlike all other known EGF-like molecules, however, the L-EGF precursor lacks a transmembrane domain, suggesting that the molecule is synthesized as a secreted peptide.

Prompted by earlier observations that injury induces an upregulation in the expression of L-EGF mRNA in the Lymnaea CNS and that purified L-EGF induces neurite outgrowth from certain types of adult neurons in vitro (Hermann et al., 2000b), we investigated whether L-EGF has a role in peripheral nerve regeneration. To test the hypothesis, we examined the effects of purified L-EGF and an EGF receptor (EGFR)-selective tyrosine kinase inhibitor on axonal regeneration of three different types of identified neurons in organ-cultured CNS.

\section{MATERIALS AND METHODS}

CNS isolation and nerve crush procedure. Adult specimens of laboratory reared Lymnaea stagnalis, 4-6 months of age, were used in all experiments. The snails were fed ad libitum with lettuce and Trout Chow (Developer Trout Ration 5D06, Purina, St. Louis, MO). All snails were kept in 701 tanks containing aerated artificial pond water of $18-20^{\circ} \mathrm{C}$ (0.26 gm/1 Instant Ocean, Aquarium Systems, Mentor, OH). Anesthesia and aseptic dissection of the CNS (including buccal ganglia) were performed as described previously (Hermann et al., 2000a). All nerves were cut as close as possible to their peripheral targets without causing any damage to their proximal parts or to the interganglionic connectives and 
commissures. After dissection, the CNSs were washed two times for 5 min in antibiotic HEPES-buffered saline composed of (in $\mathrm{mM}$ ): 51.3 $\mathrm{NaCl}, 1.7 \mathrm{KCl}, 4.1 \mathrm{CaCl}_{2}, 1.5 \mathrm{MgCl}_{2}, 5 \mathrm{HEPES}, \mathrm{pH} 7.9,150 \mu \mathrm{g} / \mathrm{ml}$ gentamycin (Sigma, St. Louis, MO). The preparations were mounted on small silicone rubber pads $\left(\sim 36 \mathrm{~mm}^{2}\right.$; RTV 616 , General Electric, Waterford, NY) using $0.1 \mathrm{~mm}$ insect pins. To avoid damage to neuronal tissue, the pins were placed only in the connective tissue sheaths surrounding the CNS. Pads, each carrying one CNS, were pinned down in pairs in a number-coded, silicone rubber-coated, saline-filled $35 \mathrm{~mm}$ polystyrene culture dish (Falcon 1008, Becton Dickinson Labware, Franklin Lakes, NJ). Subsequently, both right parietal (RPa) nerves (i.e., right internal parietal and right external parietal nerves; see Fig. 1A) were crushed with fine forceps $\sim 300-350 \mu \mathrm{m}$ distal from the CNS. To prevent inadvertent observer biases, the number-coded culture dishes were randomly assigned to different treatment groups before the RPa nerves were crushed, and all subsequent experimental procedures were performed without revealing the coding scheme. The treated CNSs were kept in antibiotic saline (1 CNS per milliliter) at room temperature in a darkened culture chamber.

Reagents, peptide reconstitution, and application. Purified and lyophilized L-EGF [purified by G. T. Nagle (University of Texas Medical Branch, Galveston, TX); see Hermann et al. (2000b) for details] was reconstituted in saline to a final concentration of $100 \mathrm{nM}$. Stocks (1 mM) of the EGFR inhibitor PD153035 (Fry et al., 1994) (Calbiochem, San Diego, CA) and the general kinase inhibitor K252a (Kamiya, Seattle, WA) were prepared in dimethylsulfoxide (DMSO; Sigma), stored at $-20^{\circ} \mathrm{C}$, and protected from light. The $1 \mathrm{~mm}$ stocks were diluted $(1000 \times)$ and added to the culture dishes such that a final concentration of $100 \mathrm{nM}$ inhibitor and the $0.1 \% \mathrm{v} / \mathrm{v}$ DMSO concentration was obtained. Control dishes contained vehicle only (DMSO $0.1 \% \mathrm{v} / \mathrm{v}$ ). All reagents were added before the nerve crush was applied (content of the dishes was unknown to the experimenter applying the crush).

Staining techniques. Axonal regeneration was principally examined by retrograde nickel lysine staining of the right internal parietal (RIP) nerve as described previously (Hermann et al., 2000a) (see also Fredman, 1987). In some cases, cells were anterogradely labeled by electrophoretically loading the cell body with a $2 \% \mathrm{w} / \mathrm{v}$ carboxyfluorescein solution (Acros, NJ) (Rao et al., 1986). The extent of regeneration (i.e., regrowth of axons across the crush site) was determined from the retrogradely labeled preparations by counting the number of stained cell bodies of three types of identified neurons known to project into the RIP nerve [right pedal dorsal 1 (RPeD1), visceral dorsal 2 and 3 (VD2/VD3), right parietal A (RPA) group motoneurons; see Fig. 1 for further explanation]. After the number of labeled cell bodies was developed and scored, the CNSs were dehydrated, defatted, mounted, and photographed for archival purposes with Kodak Tri-X 400 film (Hermann et al., 2000a). For presentation in the figures, negatives representative of each group were scanned and assembled using Photoshop 4.0.1 or Illustrator 7.0 (Adobe Systems, San Jose, CA). Contrast and brightness of individual images were adjusted to similar levels.

Data analysis and statistics. Each experiment was performed in triplicate, with each replication including $>10 \mathrm{CNSs}$ per condition. To limit biases caused by factors beyond our control (e.g., diurnal or seasonal variation, age, or endocrine or nutritional status of the animals), all experiments were performed concurrently on matched experimental and control groups comprised of animals randomly selected from the same tank. The numbers of preparations $(n)$ given in the text or figure legends reflect the total number of CNSs included under a particular condition (i.e., total of replicates).

Analysis of treatment effects in multivariate nominal data sets (i.e., labeling in the single neurons RPeD1 and VD2/VD3) was done by means of hierarchical log-linear modeling (Rodgers, 1998). This technique, which can be regarded as the equivalent of multivariate ANOVA for nominal data, analyzes the association between multiple crossclassified variates by looking for significant interactions between variates. Briefly, starting with a saturated log-linear model (i.e., all possible interaction terms), individual interaction terms that do not contribute significantly to the explanation of the observed data distribution are omitted from the model iteratively (significance of individual terms is evaluated by means of likelihood ratio $\chi^{2}$ test statistic, $G^{2}$ ). This procedure renders the most parsimonious model explaining the observed sample frequencies. For example, in analyzing the effect of L-EGF on regeneration of axonal projections of VD2/VD3 and RPeD1 into the RIP nerve, the saturated model consisted of all second-order interaction terms for the following three nominal variates: cell type (denoted as "cell type"), absence or presence of L-EGF (denoted as "treatment"), and absence or presence of retrograde labeling (denoted by "label"). Thus, in this instance the saturated model statement read cell type $\times$ treatment $\times$ label $=$ cell type $\times$ treatment + cell type $\times$ label + treatment $\times$ label Analysis of this model showed that the first two interaction terms did not contribute significantly to the observed data distribution, thus reducing the model to a most parsimonious form reading cell type $\times$ treatment $x$ label $=$ treatment $\times$ label. Other variates were included in the analysis of other data sets. Generally, treatment with kinase inhibitors or L-EGF was denoted by treatment in the model statements (see Table 1, data sets $\mathrm{B}-\mathrm{E}$ ). The duration of organ culture was denoted by "duration" in the model statement (Table 1, data set A). Analysis of associations in $2 \times 2$ cross-tabulated data was performed by means of $\chi^{2}$ tests.

Treatment effects on the number of labeled RPA somata per CNS were analyzed by means of ANOVA. In the latter case a $\sqrt{ }\left(x_{I}+1\right)$ transformation of the data was performed before analysis to correct for the right-skewness of the distributions. Frequency distributions were evaluated against the normal distribution using the Kolmogorov-Smirnov one-sample test. Means are presented with their SEM.

\section{RESULTS}

In this study we examined axonal regeneration after a crush injury to the RIP nerve (Fig. $1 A$ ). This nerve contains axons of a number of readily identifiable neurons, among them several of the cells that were included in our previous study of the trophic effects of L-EGF in vitro, i.e., RPeD1, VD2/VD3, and RPA group motoneurons. All of these neurons can be identified with certainty on the basis of the size and location of their somata in the $\mathrm{CNS}$, even in the absence of labeling by the retrograde tracer (Figs. 1, 2B). In our previous in vitro study we showed that L-EGF has a neurotrophic effect on VD2/VD3 and RPA neurons but not on RPeD1 (Hermann et al. 2000b).

\section{Validation of nerve injury model}

Because the present model has not been used in a quantitative study of this kind, we first examined a number of its properties under control conditions. First, we assessed reproducibility of the nerve crush procedure. For this purpose, RIP nerves were crushed in 10 preparations, and the nerves were then cut distally at some distance from the location of the crush and immediately backfilled with nickel-lysine. As a positive control, we backfilled uncrushed RIP nerves in 20 preparations immediately after dissection. Comparison of Figure 2, $A$ and $B$, illustrates the differences in labeling observed between the two conditions. In uncrushed preparations, backfilling the RIP nerve resulted in staining of the entire nerve and many of the neurons in the right parietal ganglion (Fig. $2 A$ ). In addition, labeled axons and cell bodies were observed in the visceral, left parietal, left and right pleural, right pedal, and right cerebral ganglia. The cell bodies of VD2/VD3 and RPeD1 were labeled in 100 and $95 \%$ of the preparations, respectively. The number of labeled RPA cells in these preparations varied over a range of 5-14, with an average of $10 \pm 0.42$ (Fig. $2 B$ ). Intriguingly, similar variability in the number of RPA neurons has been reported before (Klaassen et al., 1998), indicating that this variation is characteristic of the preparation rather than an idiosyncrasy of our retrograde tracing technique. In conclusion, these results show that backfilling the RIP nerve consistently labels the cell bodies of the neurons included in this study; i.e., the technique can be used to reliably assess axonal continuity in the RIP nerve.

Crushing the RIP nerve completely disrupted retrograde transport of the tracer across the site of injury into the CNS in all 10 preparations. In these preparations we did not observe a single labeled cell body (Fig. 2B) (note that the crush did not sever the connective tissue sheath enveloping the nerve). Thus, we conclude that crushing completely transected all axons in the RIP 

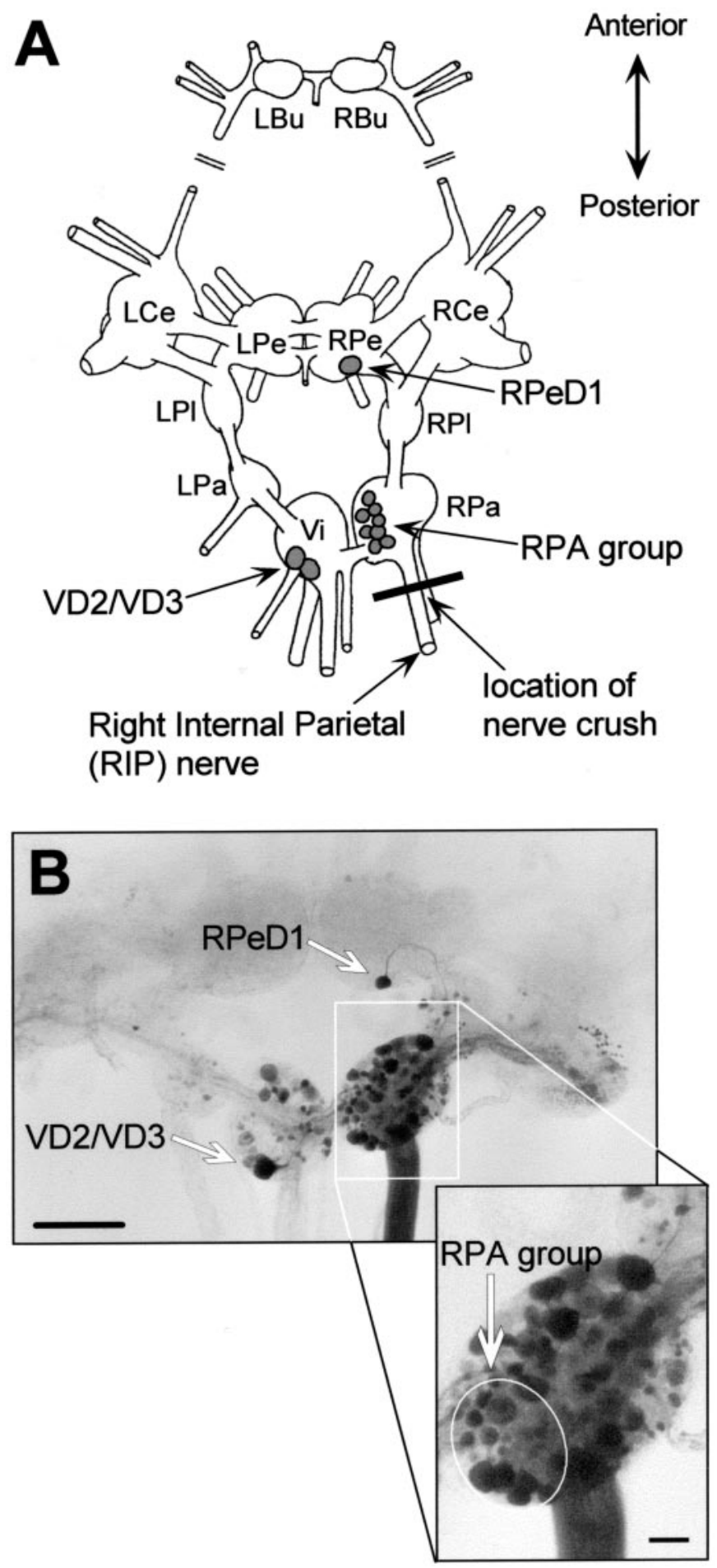

Figure 1. Experimental model and nerve injury procedure. $A$, Schematic representation of the dorsal aspect of the Lymnaea CNS with the cerebral commissure cut and cerebral ganglia folded outward. $L B u / R B u$, Left and right buccal ganglia; $L C e / R C e$, left and right cerebral ganglia; $L P e / R P e$, left and right pedal ganglia; $L P l / R P l$, left and right pleural ganglia; $L P a / R P a$, left and right parietal ganglia; $V i$, visceral ganglion; $R P e D 1$, right pedal dorsal $1 ; R P A$, right parietal A neurons; $R P D 1$, right parietal dorsal 1; $V D 2 / V D 3$, visceral dorsal 2 and 3 [nomenclature according to Benjamin and Winlow (1981)]. B, Microphotograph of a Lymnaea CNS after retrograde staining of the RIP nerve (buccal ganglia not shown). Scale bar, $500 \mu \mathrm{m}$. In this case no nerves were crushed. Backfilling the RIP nerve labeled numerous neuronal somata. Particularly, RPeD1, VD2/VD3, and the RPA group motoneurons are clearly visible (also see enlarged inset of RPa ganglion). Scale bar, $100 \mu \mathrm{m}$.
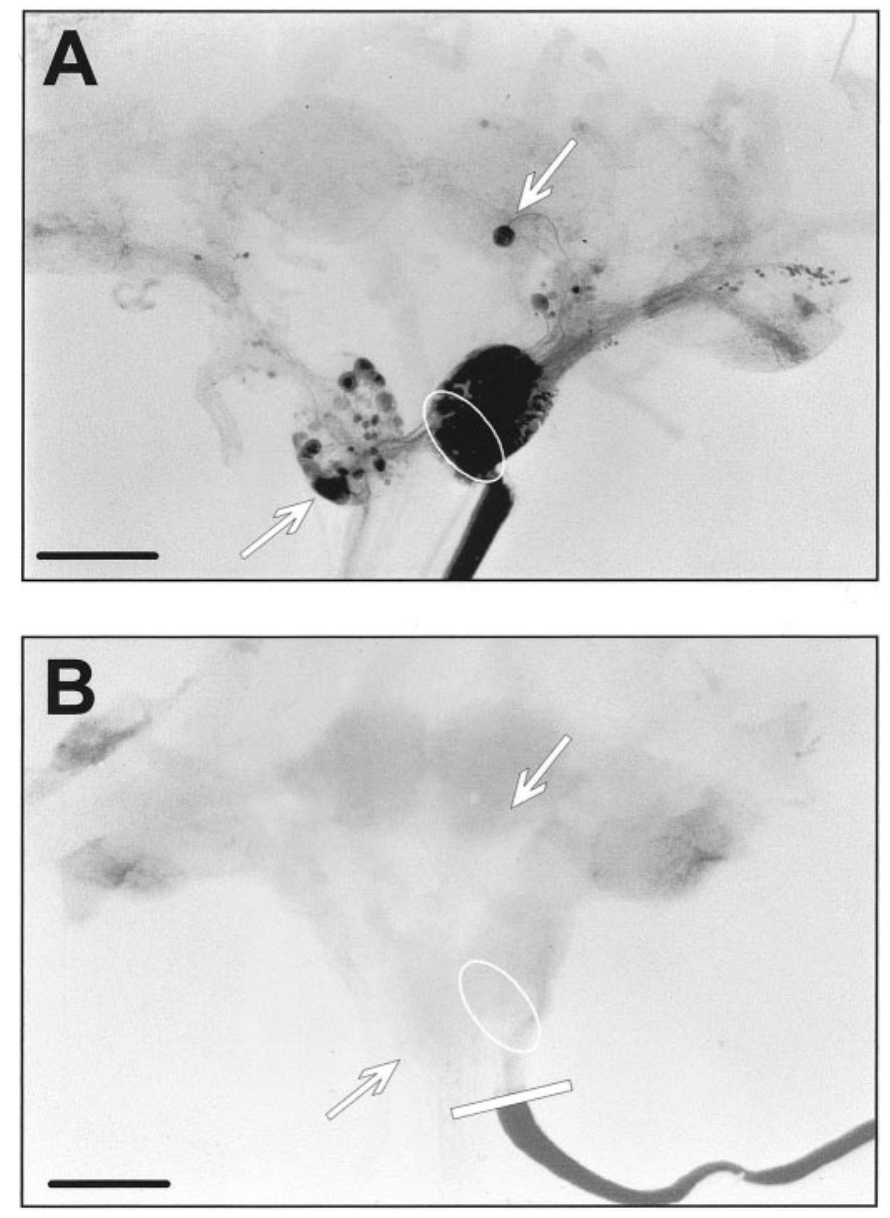

Figure 2. Effect of proximal crush and organ culture on axonal projections into the RIP nerve. $A$, Microphotograph of a CNS (dorsal view, with the cerebral commissures cut) that was retrograde labeled immediately after isolation without crushing the RIP nerve. Scale bar, $500 \mu \mathrm{m}$. Numerous labeled neuronal somata are seen in several ganglia, including those of RPeD1, VD2/VD3, and several RPA neurons. B, Microphotograph of a CNS (dorsal view, with the cerebral commissures cut) in which the RIP nerve was backfilled with nickel-lysine immediately after the nerve was crushed. No neuronal somata are labeled in this preparation, indicating that axonal projections projecting into the RIP nerve were completely severed (note that the tracer is not transported across the crush). Scale bar, $500 \mu \mathrm{m}$.

nerve. Note that despite the absence of staining in the proximal part of the RIP nerve and the CNS, the distal part of the nerve was densely stained. This indicates that the tracer was taken up and retrogradely transported by the remaining distal axon stumps; i.e., the failure to label cells in the CNS did not result from inadequate internalization of the tracer. Loading failures could be easily recognized by a transparent RIP nerve trunk and occurred infrequently ( $2 \%$ of all preparations); these cases were excluded from the study.

Next we verified whether the axons of neurons that project into the RIP nerve remained stable for the duration of our experiments. For this purpose two additional sets of CNS were dissected. One was kept in culture for $2 \mathrm{~d}(n=22)$, and the other was cultured for $7 \mathrm{~d}(n=17)$ before their RIP nerves were backfilled. Comparison of these two data sets with those from acutely backfilled preparations showed that RPeD1, VD2/VD3, and RPA neurons were labeled with similar efficiency under all three conditions (Fig. $3 A$ ). In fact, VD2/VD3 were labeled in $100 \%$ of 

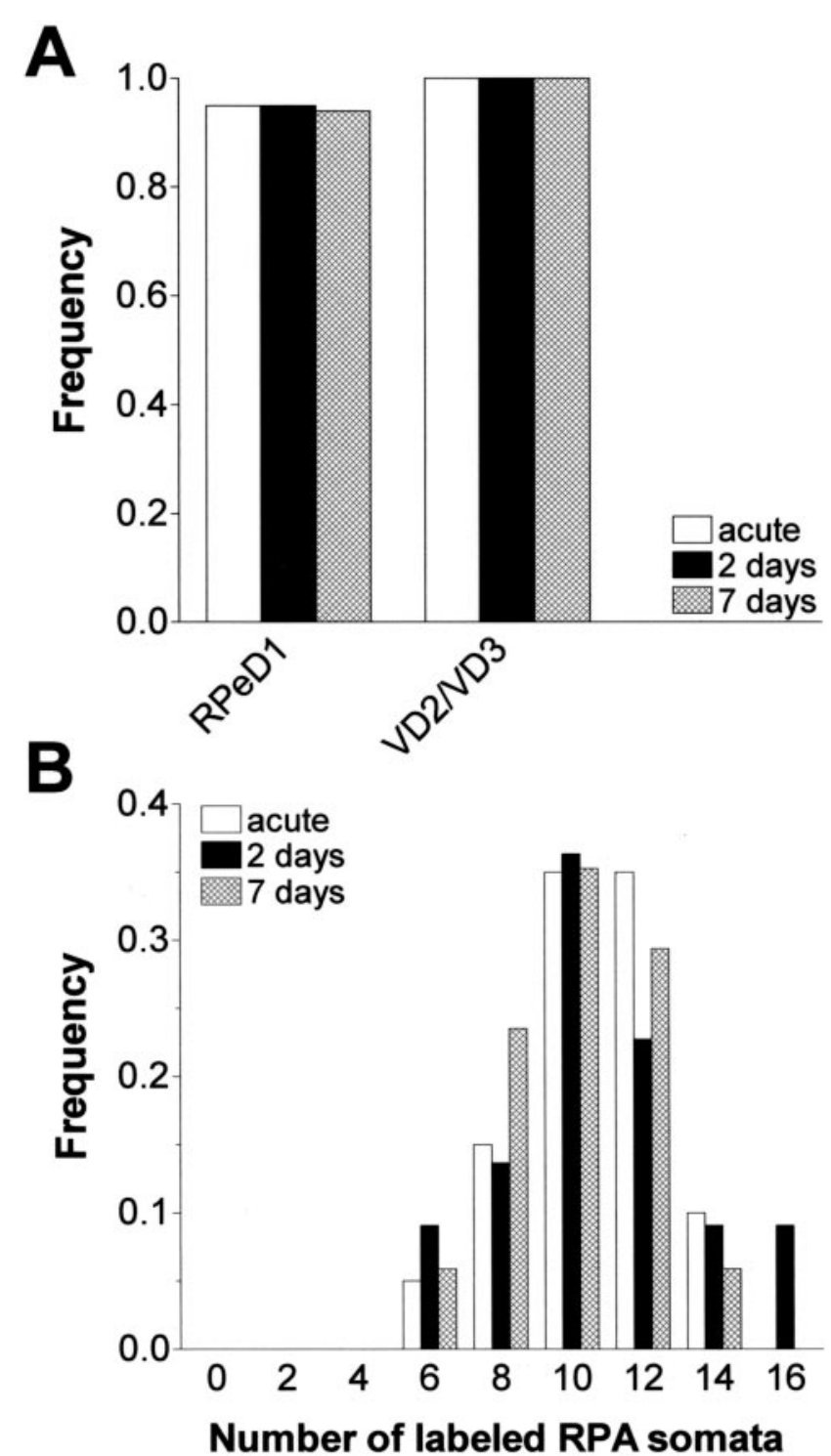

Figure 3. Stability of RIP nerve axonal projections in organ culture. $A$, Frequency of preparations in which RPeD1 and VD2/VD3 were retrograde labeled by backfilling the RIP nerve either immediately (acute), after $2 \mathrm{~d}$ in culture ( 2 days), or after $7 \mathrm{~d}(7$ days) in culture. Note that there was no difference in the frequency of labeled RPeD1 and VD2/VD3 somata between the three conditions. $B$, Frequency distribution of labeled RPA somata per CNS in preparations in which the RIP nerve was backfilled either immediately (acute; $n=20$ ), after $2 \mathrm{~d}$ in culture ( 2 days), or after $7 \mathrm{~d}(7$ days $)$ in culture. Note that the data are distributed very similarly in all three cases, with a range of 5 to 15 and a median value of 10 labeled RPA somata per CNS (bin width $=2$ ).

the cases, whereas an unlabeled RPeD1 was observed only once in each of the three data sets.

Regarding RPA neurons, the average number of labeled somata was very similar in acutely backfilled preparations and those backfilled after 2 or $7 \mathrm{~d}(9.9 \pm 0.42,10.5 \pm 0.61$, and $9.6 \pm 0.46$, respectively; $\left.F_{(2,56)}=1.611 ; p=0.21\right)$. Moreover, the data in these three groups were distributed similarly (range 5-16) and were not significantly different from the normal distribution (Fig. $3 B$ ). This observation leads to three important conclusions. First, it shows that the number of RPA neurons that project axons into the RIP nerve is variable but never $<5$. Second, organ culture does not cause significant changes in the shape of the data distribution; i.e., there is no net addition or loss of RPA projections into the RIP nerve for a period of at least $7 \mathrm{~d}$. Third, retrograde labeling of RPA neurons can be done with similar efficiency whether done acutely or after 2 or $7 \mathrm{~d}$ in culture. Taken together, these results show that dissection and culturing of the CNS does not compromise the stability of the peripheral projections of all three types of neurons included in this study. Thus, we conclude that the organ-cultured CNS provides a stable platform for analyzing axonal regeneration for periods of $<7 \mathrm{~d}$.

\section{Axonal regeneration: time course}

To optimally assess the effect of experimental intervention on axonal regeneration, data should be sampled during active neurite elongation. Estimates of neurite elongation rates in the molluscan CNS suggest that regenerating axons should be able to re-extend over considerable distance in the RIP nerve within $2 \mathrm{~d}$ (Allison and Benjamin, 1985; Kruk and Bulloch, 1992; Hermann et al., 2000a). We tested whether $2 \mathrm{~d}$ would suffice to obtain optimal regeneration by comparing the number of labeled neurons in acutely backfilled control preparations with CNSs that received a nerve crush and were cultured for either 2 or $7 \mathrm{~d}$.

After $2 \mathrm{~d}$ of culture, RPeD1 or VD2/VD3 was labeled in $>30 \%$ of the cases; i.e., in nearly one-third of the preparations these cells had extended their neurites across the crush site (Fig. $4 A)(n=89)$. In the same group of CNSs, one or more labeled RPA somata were counted in $78 \%$ of the preparations (Fig. $4 B$ ). The mean number of labeled RPA neurons in this group was significantly lower, however, than in the controls $(5.5 \pm 0.48, n=$ 89 vs $\left.10 \pm 0.42, n=20 ; F_{(1,102)}=17.86 ; p<0.001\right)$. Figure $4 B$ also illustrates that the corresponding data distribution was strongly skewed to the right, implying that regeneration was incomplete in most preparations rather than totally absent in one subset and nearly complete in another (the latter would have resulted in a bimodal distribution). Also, the observation that high numbers of labeled RPA somata (i.e., >12) were counted in a small percentage of preparations suggests that regeneration of RPA axons into the RIP nerve was complete or nearly complete in some cases.

Comparison of the data sets shows that the incidence of labeled neurons only marginally improved by extending the incubation period (Fig. $4 A, B$ ). Consistent with this observation, log-linear analysis of the RPeD1 and VD2/VD3 data indicated no significant association between labeling and cell type or between labeling and incubation duration (Table 1, data set A). Moreover, although the mean number of labeled RPA somata per CNS after $7 \mathrm{~d}$ in culture was slightly higher than after $2 \mathrm{~d}(7.1 \pm 0.60, n=$ 50 , vs $5.5 \pm 0.48)$, it remained significantly below the control value $\left(10 \pm 0.42 ; F_{(1,89)}=16.51 ; p<0.001\right)$. The major difference between the two data sets was a reduction in the percentage of the preparations in which no RPA somata were labeled in the $7 \mathrm{~d}$ set (Fig. 4B).

It could be argued that the less than optimal labeling observed in the regenerating preparations is caused by a failure of the injured, regenerating axons to transport the retrograde tracer to the cell body. To examine this possibility, we filled RPeD1 cell bodies with the anterograde tracer carboxyfluorescein in preparations that had received a nerve crush $2 \mathrm{~d}$ earlier. Inspection of these preparations revealed that regenerating RPeD1 neurites had extended across the injury zone in $35 \%$ of the cases $(n=17)$. In the remaining $65 \%$ of the preparations, neurites had failed to invade the injury zone. Thus, anterograde and retrograde labeling techniques rendered similar scores in this regeneration model 

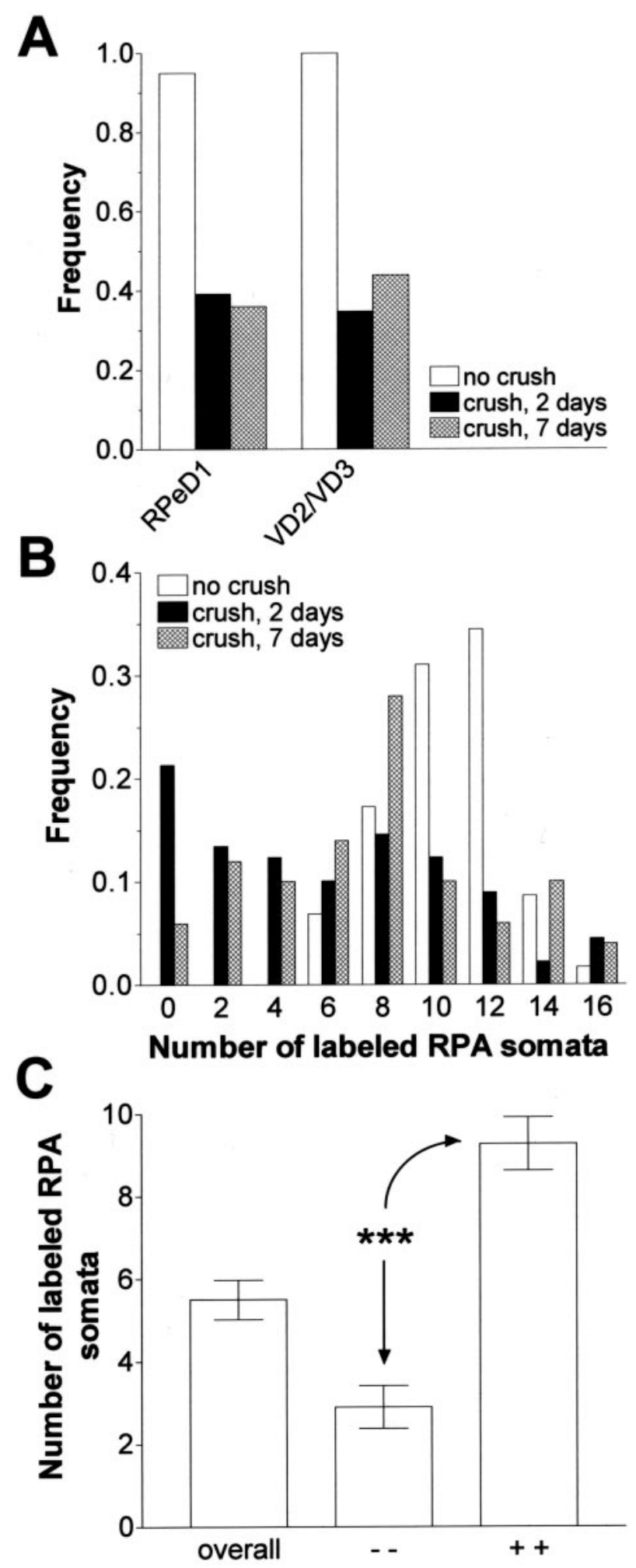

Figure 4. Regeneration of RIP axons in isolated CNS cultured in saline. $A$, Frequency of preparations in which RPeD1 and VD2/VD3 were retrograde labeled by backfilling the RIP nerve immediately after dissection of the CNS without nerve crush (no crush) or by backfilling the RIP nerve in crushed preparations after $2 \mathrm{~d}$ (crush, 2 days) or $7 \mathrm{~d}$ (crush, 7 days) in culture, respectively. Note that RPeD1 and VD2/VD3 are labeled in $<40 \%$ of the preparations after $2 \mathrm{~d}$ in culture and that for both cell types this proportion did not significantly improve by keeping the preparations in culture for $7 \mathrm{~d}$ in culture. $B$, Frequency distribution of the number of retrograde-labeled RPA somata per CNS in preparations that
(35 and $37 \%$, respectively), indicating that there is no reason to assume that the latter technique incorrectly estimates the extent of regeneration.

We also investigated whether individual neurons regenerated their axons independently or in a coordinated manner. We found a prominent coincidence in labeling of RPeD1 and VD2/VD3 within preparations. In $87 \%$ of the preparations cultured for $2 \mathrm{~d}$ $(n=89)$, either both or neither RPeD1 and VD2/VD3 somata were labeled (38 and 49\%, respectively), leaving $13 \%$ of preparations in which only one of these cell types was labeled. Statistical analysis confirmed that axonal regeneration in both cell types coincided more frequently than expected on a random basis $\left(\chi_{(1)}^{2}=45.024 ; p<0.001\right)$. This association between labeling in different cell types also appeared to apply to RPA neurons (Fig. $4 C$ ). The average number of labeled RPA somata was more than three times higher in preparations in which both RPeD1 and VD2/VD3 somata were labeled in comparison with those in which neither of these two cell types was labeled (Student's $t$ test $=7.427$; df $=74 ; p<0.001$ ).

Taken together, these results show that in the organ-cultured CNS, axonal regeneration of all three types of neurons proceeded rapidly during the first $2 \mathrm{~d}$ after injury and slowed down considerably thereafter. Therefore, in the remainder of the study we adopted $2 \mathrm{~d}$ as the routine duration of our assays. The data also show that considerable variation exists in the rate and extent of axonal regeneration between individual preparations; i.e., even after $7 \mathrm{~d}$ in culture some do not regenerate a single RPA axon, whereas others regenerate the full complement of 15 axons within $48 \mathrm{hr}$ after the RIP nerve is crushed. Moreover, the results support the view that axonal regeneration in the organ-cultured CNS occurs in a coordinated manner.

\section{L-EGF promotes axonal regeneration}

To investigate whether L-EGF affects axonal regeneration, 83 preparations were cultured for $2 \mathrm{~d}$ in either saline $(n=44)$ or saline plus L-EGF $(n=39)$. In these experiments L-EGF was used at a concentration of $100 \mathrm{~nm}$, a dose previously shown to have a near maximal neurotrophic effect on RPA neurons in vitro (Hermann et al., 2000b). Figure 5, $A$ and $B$, illustrates the key finding of the present study. These figures show the dramatic difference in labeling observed in control preparations and preparations treated with L-EGF. The frequency of labeling of $\mathrm{RPeD} 1$ and VD2/VD3 significantly increased from $\sim 30 \%$ to nearly $60 \%$ in the presence of the peptide (Fig. $5 C$; Table 1, data set B). Intriguingly, although our previous study revealed differences in the in vitro responsiveness of RPeD1 and VD2/VD3 to L-EGF, statistical analysis of the present data indicated that L-EGF indiscriminately facilitated axonal regeneration of both cell types (Table 1 , data set B).

Treatment with L-EGF also enhanced the number of labeled RPA neurons. On average, the number of labeled cells increased from $3.8 \pm 0.55$ to $6.8 \pm 0.64$ in L-EGF $\left(F_{(1,81)}=13.161 ; p<\right.$ $0.0001)$. This effect involved a substantial reduction in the percentage of preparations with fewer than five labeled RPA somata

$\leftarrow$

were backfilled immediately after dissection without crushing the RIP nerve (no crush) and preparations in which the RIP nerve was crushed and backfilled after $2 \mathrm{~d}$ in culture (crush, 2 days) and $7 \mathrm{~d}$ (crush, 7 days) in culture, respectively. $C$, Average number of labeled RPA somata in the total $2 \mathrm{~d}$ data set (overall), a subset of the $2 \mathrm{~d}$ data in which neither RPeD1 nor VD2/VD3 was labeled $(--)$, and a subset of the $2 \mathrm{~d}$ data in which both RPeD1 and VD2/VD3 were labeled $(++)$. ${ }^{* * *} p<0.001$. 
Table 1. Summary of log-linear analysis of RPeD1 and VD2/VD3 regeneration scores observed in the five different experiments comprising this study (data sets A-E)

Data set
A, Duration
B, L-EGF only
C, PD153035 plus L-EGF
D, PD153035 only
E, K252a only

Most parsimonious log-linear model

\begin{tabular}{|c|c|}
\hline Likelihood ratio $\chi^{2}$ & $p$ \\
\hline$G_{(5)}^{2}=1.226$ & 0.94 \\
\hline$G_{(6)}^{2}=0.6218$ & 0.99 \\
\hline$G_{(6)}^{2}=9.985$ & 0.24 \\
\hline$G_{(6)}^{2}=2.260$ & 0.81 \\
\hline$G_{(3)}^{2}=0.5128$ & 0.92 \\
\hline
\end{tabular}

Shown are the most parsimonious log-linear models with their associated likelihood ratio $\chi^{2}$ (degrees of freedom in subscript) and $p$ value. [Contrary to most other statistical techniques, high values of the test statistic combined with a significant $p$ value indicate that the evaluated model does not adequately explain the sample frequencies. Thus, a model adequately describing the data distribution is associated with a low value of $G^{2}$ combined with a nonsignificant $p$ value; i.e., $p>0.05$ (Rodgers, 1998 ).]

Figure 5. Effect of L-EGF on regeneration of crushed RIP axons. $A, B$, Microphotographs of isolated CNSs (dorsal view, with the cerebral commissures cut) that were cultured for $2 \mathrm{~d}$ after receiving a crush to the RIP nerve in saline $(A)$ or in saline plus $100 \mathrm{~nm}$ L-EGF $(B)$. Scale bar, $500 \mu \mathrm{m}$. Comparison of both photographs illustrates that the number of labeled neuronal somata was dramatically enhanced in the presence of L-EGF. $C$, Frequency of preparations in which RPeD1 and VD2/ VD3 were retrograde labeled after $2 \mathrm{~d}$ in the presence of $100 \mathrm{nM}$ L-EGF (saline $+L-E G F)$ and without (saline only). Treatment with L-EGF significantly enhanced the proportion of preparations that extended axons from RPeD1 and VD2/VD3 into the damaged RIP nerve. $D$, Frequency distribution of labeled RPA somata per CNS in preparations that were cultured for $2 \mathrm{~d}$ in the absence (saline only) and presence of $100 \mathrm{~nm}$ L-EGF (saline $+L-E G F$ ) (bin width $=2$ ). Treatment with L-EGF significantly enhanced regeneration of RPA axons projecting into the RIP nerve.
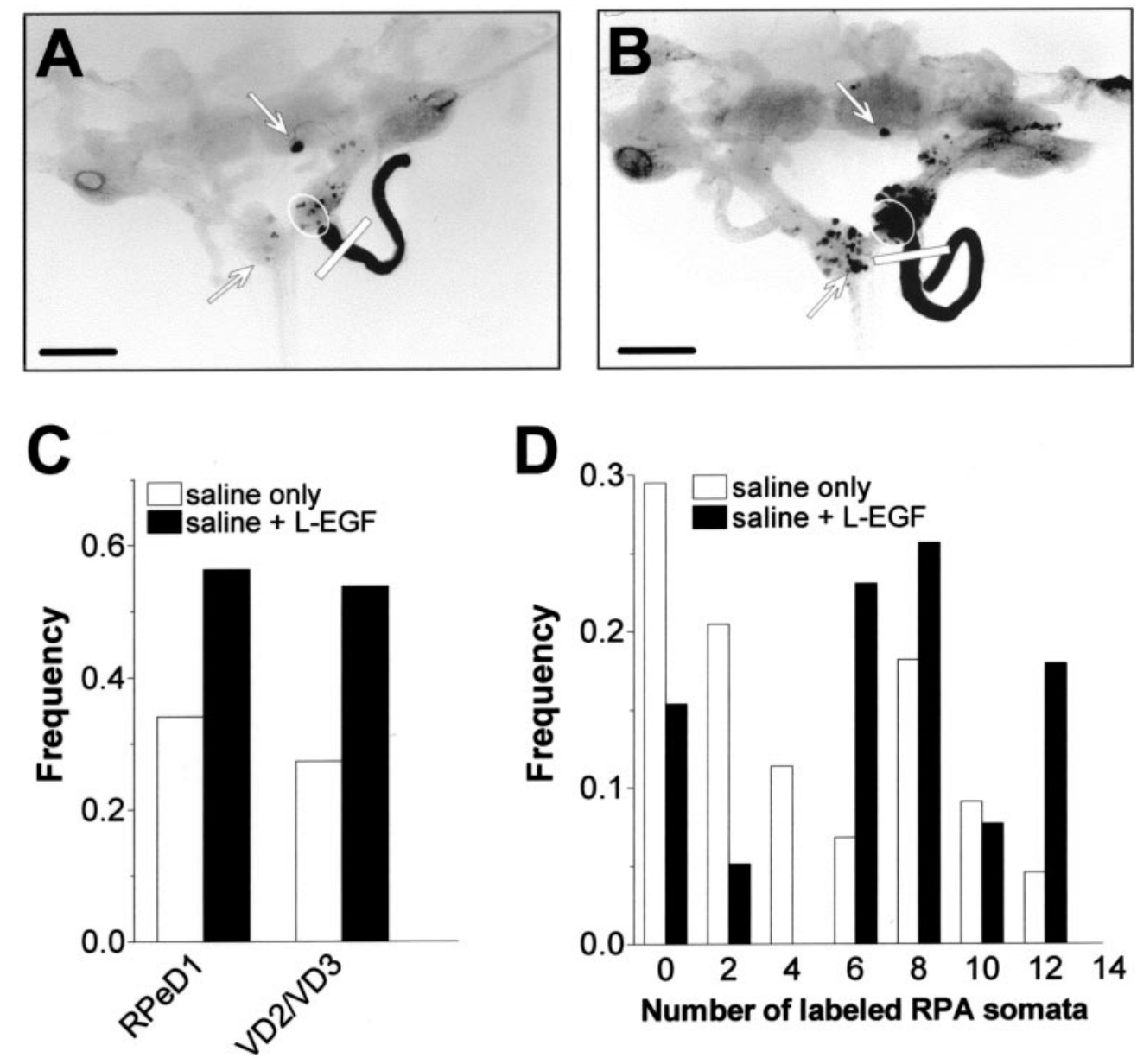

(from 61 to $20 \%$ of preparations) and a global increase in the representation of preparations over the entire range of the data distribution (Fig. 5D). This indicates that L-EGF enhanced axonal regeneration in most preparations rather than boosting the number of labeled RPA neurons to high levels in a few preparations.

We also analyzed the association between labeling in the different cell types in this data set. This analysis supported the notion that axonal regeneration of RPeD1 and VD2/VD3 within preparations occurs in coordinated manner, with or without L-EGF. Specifically, we found matching labels (i.e., both labeled or both unlabeled) in $93 \%$ of the control preparations and $72 \%$ of the preparations cultured in the presence of L-EGF, respectively $\left(\chi_{(1)}^{2}=31.90, p<0.001\right.$, and $\chi_{(1)}^{2}=7.24, p<0.01$, respectively). Likewise, the mean number of labeled RPA somata was more than two times higher in the preparations in which both RPeD1 and VD2/VD3 were labeled after treatment with L-EGF (3.7 \pm 1.14, $n=11$ vs $9.9 \pm 0.53, n=18$ ).

In conclusion, these results demonstrate that that L-EGF enhances axonal regeneration in the organ-cultured CNSs of all three cell types included in the study. The observation that L-EGF facilitated axonal regeneration in RPeD1, a cell incapable of responding to L-EGF with neurite outgrowth in vitro, as well as in VD2/VD3 and RPA neurons suggests that some of the neurotrophic effects of the peptide in the nervous system are mediated indirectly.

\section{Inhibition of L-EGF actions by the specific EGFR inhibitor, PD153035}

To test the involvement of an EGFR in the effects of L-EGF, we examined the actions of the specific EGFR tyrosine kinase inhib- 
itor, PD153035. Previously, we showed that this compound selectively inhibits both L-EGF-induced and human recombinant EGF-induced neurite outgrowth in Lymnaea neurons in vitro (Hermann et al., 2000b).

Two groups of CNSs were dissected, and their RIP nerves were crushed and cultured for $2 \mathrm{~d}$. The control group was cultured in saline containing $100 \mathrm{nM}$ L-EGF only $(n=32)$; the second group was treated with $100 \mathrm{~nm}$ L-EGF plus $100 \mathrm{~nm}$ PD153035 $(n=31)$. In the controls (i.e., L-EGF only), we observed extensive labeling of cell bodies throughout the visceral, right parietal, and left pleural ganglia, and some labeling in the right cerebral and right parietal ganglia. In general, the number of labeled cell bodies was lower in preparations treated with PD153035. Specifically, the frequency of preparations in which RPeD1 and VD2/ VD3 were labeled was significantly reduced after treatment with PD153035 (Fig. 6A; Table 1, data set C). Note that the absence of significant association between cell type and treatment in the model statement indicates that PD153035 suppressed axonal regeneration of both RPeD1 and VD2/VD3, an observation consistent with the previous conclusion that L-EGF indiscriminately facilitates regeneration of both cell types.

With regard to RPA neurons, in comparison with the control group, the mean number of labeled somata was significantly lower in preparations treated with both L-EGF and PD153035 (6.4 \pm 0.65 vs $\left.3.5 \pm 0.54 ; F_{(1,61)}=11.461 ; p<0.001\right)$. Figure $6 B$ illustrates that this effect was mainly because of a substantial increase in the proportion of preparations with no labeled RPA somata.

Labeling of individual cells within preparations again showed a statistically significant association. In the control group, RPeD1 and VD2/VD3 were both labeled or unlabeled in $87 \%$ of the preparations $\left(\chi_{(1)}^{2}=18.29 ; p<0.001\right)$. In preparations treated with L-EGF plus PD153035, matching labels were found in $77 \%$ of the preparations $\left(\chi_{(1)}^{2}=5.11 ; p=0.024\right)$. Moreover, the mean number of labeled RPA neurons in the preparations with labeled RPeD1 and VD2/VD3 was significantly higher than in the preparations in which neither of these two cell types was labeled $(7.5 \pm 1.76, n=4$ vs $2.15 \pm 0.51, n=20 ; t=3.928 ;$ df $=22 ; p<$ $0.001)$. Taken together these results demonstrate that treatment with PD153035 counteracted the effect of L-EGF on axonal regeneration.

\section{A role for endogenously released L-EGF in axonal regeneration?}

Our previous work has shown that L-EGF mRNA expression is upregulated after CNS injury (Hermann et al., 2000b). Therefore, we examined whether activation of an EGFR by endogenously released L-EGF (or one of its family members) contributes to axonal regeneration in the Lymnaea CNS. To this end, axonal regeneration was analyzed in CNS preparations cultured for $2 \mathrm{~d}$ in either saline $(n=45)$ or saline plus $100 \mathrm{~nm}$ PD153035 $(n=43)$ without adding purified L-EGF to the media. Generally, labeling was less extensive in the preparations treated with PD153035 (Fig. 7). With regard to VD2/VD3 and RPeD1, analysis of the data indicated a significant interaction between the occurrence of labeling and treatment with the inhibitor, but no significant interaction between cell type and the occurrence of labeling (Table 1, data set D), implying that PD153035 significantly reduced the incidence of labeling of RPeD1 and VD2/ VD3 regardless of cell type (Fig. $7 A$ ). The average number of labeled RPA somata was significantly lower in preparations treated with PD153035 $(7.1 \pm 0.71$ in saline vs $4.7 \pm 0.62$ in saline
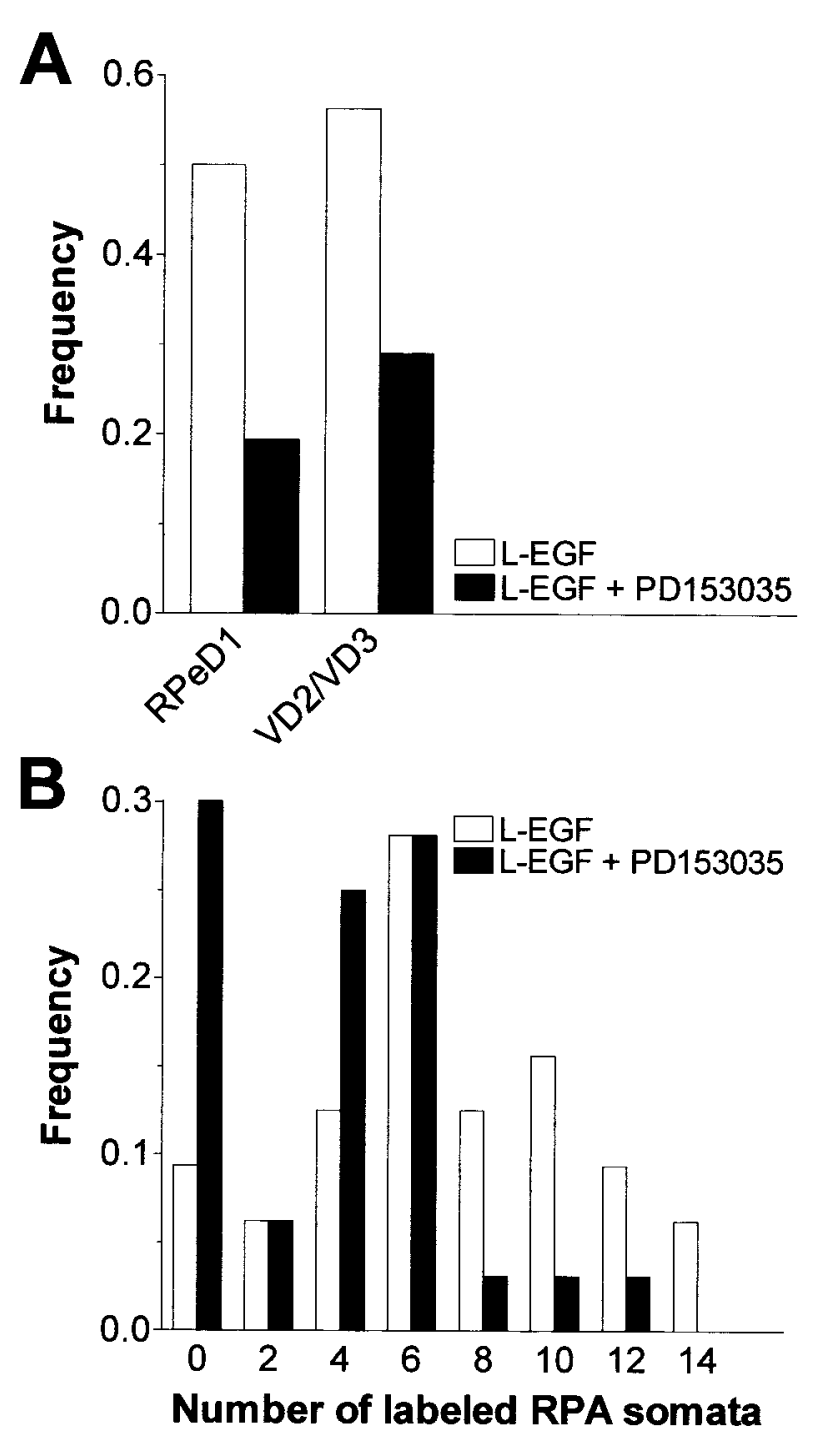

Figure 6. Effect of the specific EGF tyrosine kinase receptor inhibitor PD153035 on L-EGF-enhanced axonal regeneration after nerve injury. $A$, Frequency of preparations in which RPeD1 and VD2/VD3 were retrograde labeled in injured preparations after $2 \mathrm{~d}$ in the presence of $100 \mathrm{~nm}$ L-EGF (L-EGF) or $100 \mathrm{~nm}$ L-EGF plus $100 \mathrm{~nm}$ PD153035 (L-EGF + $P D 153035)$. Significantly fewer preparations with labeled RPeD1 or VD2/ VD3 somata were observed in the presence of PD153035. B, Frequency distribution of labeled RPA somata per CNS in injured preparations that were cultured for $2 \mathrm{~d}$ in saline plus $100 \mathrm{nM}$ L-EGF $(L-E G F)$ or $100 \mathrm{~nm}$ L-EGF plus $100 \mathrm{~nm}$ PD153035 (L-EGF + PD153035) (bin width $=2$ ). Compared with controls, the number of labeled RPA somata was significantly lower in preparations treated with L-EGF + PD153035.

plus PD153035; $\left.F_{(1,86)}=6.183 ; p=0.015\right)$, an effect mainly attributable to a reduction in the percentage of preparations with more than eight labeled cell bodies (Fig. 7B).

Although a highly significant association between labeling in RPeD1 and VD2/VD3 was observed again in the control group (matching labels in 78\% of the preparations; $\chi_{(1)}^{2}=13.34 ; p<$ $0.001)$, the association between labeling in both cell types after treatment with PD153035 was not as clear-cut as in the previous sets. Because of the imbalance in the data distribution (i.e., both RPeD1 and VD2/VD3 were unlabeled in five-sixths of the 30 preparations), statistical analysis of this data was regarded as inconclusive. The mean number of labeled RPA neurons, however, was significantly different in both sets of preparations $(t=$ 

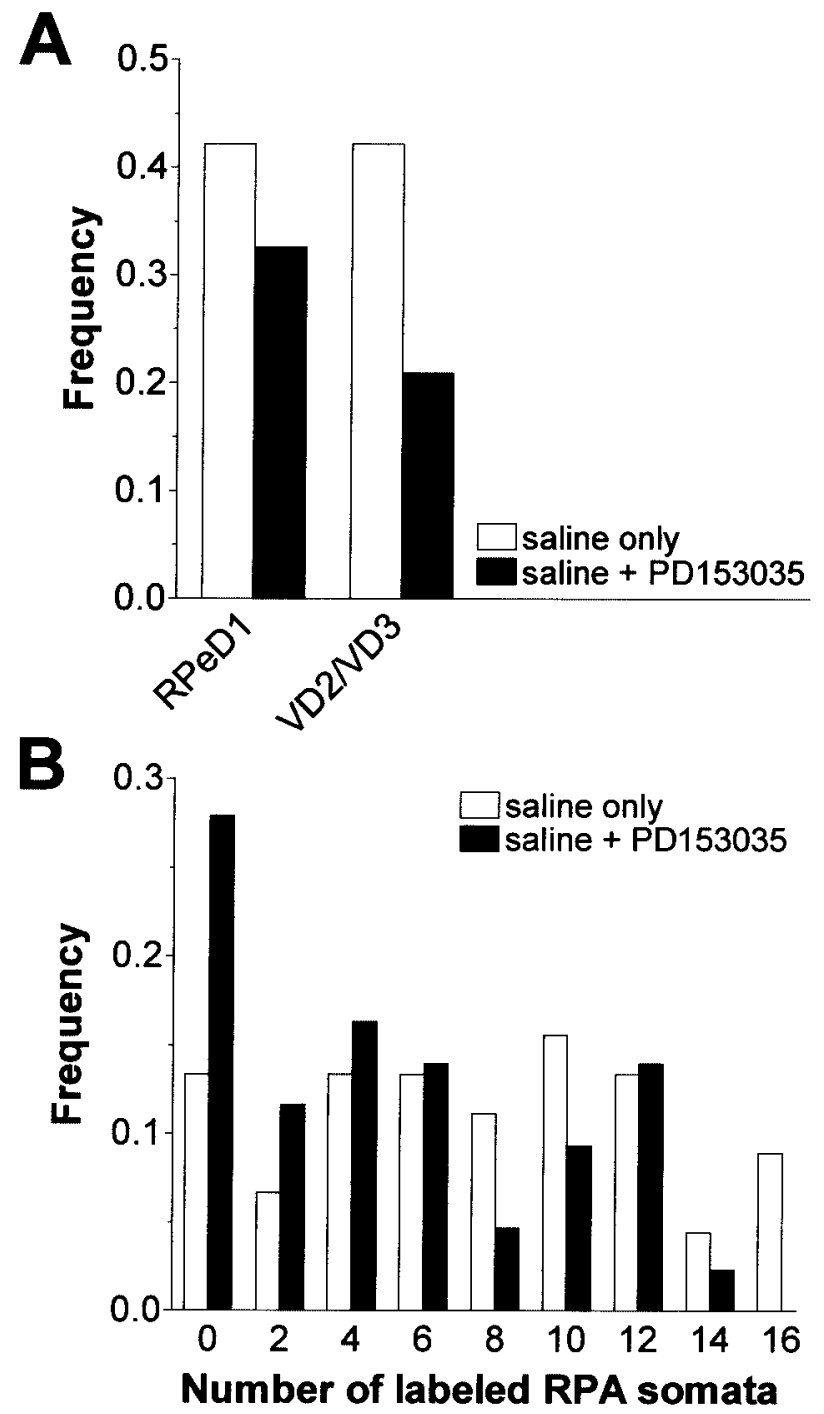

Figure 7. Effect of the specific EGF tyrosine kinase receptor inhibitor PD153035 on axonal regeneration after RIP nerve crush in saline without L-EGF supplementation. $A$, Frequency of preparations in which RPeD1 and VD2/VD3 were retrograde labeled by backfilling the RIP nerve after $2 \mathrm{~d}$ in culture in saline (saline only) or saline plus $100 \mathrm{~nm}$ PD153035 (saline + PD153035). Significantly fewer preparations with labeled RPeD1 or VD2/VD3 were observed in the presence of the kinase inhibitor. $B$, Frequency distribution of labeled RPA somata per CNS in preparations that were cultured for $2 \mathrm{~d}$ in saline plus $100 \mathrm{nM}$ L-EGF (saline only) or saline plus $100 \mathrm{~nm}$ PD153035 (saline + PD153035) (bin width $=2$ ). Compared with controls, treatment with PD153035 caused a significant reduction in number of labeled RPA somata.

6.481; df $=28 ; p<0.001 ; 11.4 \pm 0.51, n=5$ in preparations with labeled RPeD1 and VD2/VD3, and $2.0 \pm 0.63, n=25$ in preparations without labeled RPeD1 and VD2/VD3).

To complement the PD153035 data with another independent data set, we also examined the actions of a broad spectrum kinase inhibitor, K252a, on axonal regeneration (K252a inhibits tyrosine kinases as well as serine/threonine kinases) (Ruegg and Burgess, 1989; Knüsel and Hefti, 1992). We showed previously that $100 \mathrm{~nm}$ K252a completely inhibits in vitro neurite outgrowth in the presence of L-EGF-, human EGF-, or CNS-conditioned medium (Hermann et al., 2000b). Sixty-four preparations received RIP nerve crushes and were cultured for $2 \mathrm{~d}$ in either saline or saline plus $100 \mathrm{~nm} \mathrm{K252a}$. In the presence of the inhibitor, the percent- age of labeled RPeD1 was significantly reduced from 39 to $13 \%$ and that of VD2/VD3 dropped from 33 to $6 \%$ (Table 1, data set E). The mean number of labeled RPA neurons was reduced by $>55 \%$ to $2.1 \pm 0.38$ in the presence of the inhibitor $(t=2.905$; $p<0.01 ; \mathrm{df}=62)$. In conclusion, K252a appears to reduce axonal regeneration to a greater extent than PD153035, a notion consistent with the broader activity spectrum of this compound.

\section{DISCUSSION}

We examined whether L-EGF, an epidermal growth factor homolog isolated from the pond snail L. stagnalis, promotes axonal regeneration in the CNS. Our main findings can be summarized as follows. (1) Treatment of organ-cultured CNSs for $48 \mathrm{hr}$ with submicromolar doses of purified L-EGF enhances axonal regeneration of three types of identified neurons (RPeD1, VD2/VD3, and RPA) by $>65 \%$. (2) The therapeutic effect of L-EGF on axonal regeneration was antagonized by the selective EGFR tyrosine kinase inhibitor, PD153035. (3) Both PD153035 and K252a significantly reduced baseline axonal regeneration of the identified neurons in this study, suggesting that EGFR ligands generated within the CNS contribute to its injury response. (4) Axonal regeneration of the different neurons included in this study is highly coordinated, both with and without L-EGF treatment.

\section{EGF and related molecules in axonal regeneration}

The present results complement our previous observation that the expression of L-EGF mRNA is upregulated after CNS injury (Hermann et al., 2000b). Although other peptides with in vitro neurotrophic activity have been identified in Lymnaea (Kits et al., 1990; Fainzilber et al., 1996), we provide the first experimental support for neurotrophic actions of an endogenous growth factor in the Lymnaea CNS.

Our results show that interesting parallels exist between the injury response of the molluscan and mammalian nervous systems, but they also reveal an intriguing difference. In both cases, EGF homologs can have neuroprotective or neurotrophic effects in vitro (Morrison et al., 1988; Kenigsberg and Mazzoni, 1995; Hermann et al., 2000b; Kimpinski and Mearow, 2001). Although our study demonstrates that L-EGF enhances peripheral nerve regeneration in Lymnaea, in vivo data in support of a similar effect of EGF homologs in the mammalian peripheral nervous system (PNS) are not available. Still, evidence generally points toward a role of the EGF family and its receptors in the injury response of the mammalian nervous system (Yamada et al., 1997; Xian and Zhou, 2000). For instance, focusing on the PNS, the part of the mammalian nervous system most analogous to the snail nervous system, it has been shown that peripheral nerve damage upregulates the expression of the EGFR (ErbB) and several of its ligands (Toma et al., 1992; Xian and Zhou, 1999). However, there is no convincing functional data showing that EGF or any of its homologs enhances peripheral nerve generation (Dubuisson et al., 1993). The reason for this lack of functional data regarding EGF homologs in vertebrates is unclear, but may reflect the extensive potential for ligand redundancy and receptor promiscuity characterizing the EGF/ErbB family signaling network in mammals (Riesse and Stern, 1998; Xian and Zhou, 2000). Although there may be more than one EGFR ligand in Lymnaea, we have identified only one to date: a simple single-domain EGF-like molecule that is similar to the mature forms of both mammalian EGF and TGF- $\alpha$ (Hermann et al. 2000b). This suggests that the gastropod equivalent of the mammalian EGF/ErbB signaling network may 
be simpler and provide fewer opportunities for ligand substitution and receptor saturation. Parenthetically, in analogy with our study, reagents such as PD153035 could be used to test the role of EGFR activation in peripheral nerve regeneration of mammals.

It is conceivable that pharmacokinetic parameters contribute to the differences in EGF responsiveness of the Lymnaea and mammalian preparations. Obviously, the physicochemical parameters governing the diff usion of peptides in the mammalian in vivo peripheral nerve injury assay are very different from those in the Lymnaea CNS organ culture system where the preparation is bathed in a large excess of peptide containing saline. Hence, the exchange of molecules between the injured nerve and the bulk of the extracellular compartment will likely be much more unrestricted in the latter preparation. It could be argued that by washing out locally generated EGFR ligands we uncovered responsiveness of the Lymnaea preparation for exogenously applied L-EGF.

Last but not least, it is well known that the capacity of the molluscan nervous system to restore damage far exceeds that of the mammalian nervous system (Janse et al., 1979, 1986; Allison and Benjamin, 1985; Moffett, 1995; Hermann et al., 2000a). Therefore, we cannot dismiss the possibility that the present therapeutic effects of L-EGF on axonal regeneration in the Lymnaea nervous system and the apparent absence of such an effect in mammals is a reflection of a phylogenetically older injury response mechanism that became obsolete (or was overruled by other requirements) during mammalian phylogeny.

\section{Site of action of L-EGF in the organ-cultured Lymnaea nervous system: all or none, direct and indirect actions?}

The opportunity to identify individual neurons within the Lymnaea CNS allowed us to address another important issue: does axonal regeneration of individual neurons occur independently or in a coordinated manner? The answer to this question provides clues to the relative importance of mechanisms operating on individual neurons versus those that affect all neurons. Our data indicate that axonal regeneration of $\mathrm{VD} 2 / \mathrm{VD} 3, \mathrm{RPeD} 1$, and RPA neurons within preparations occurs concurrently with a significantly higher frequency than one would expect to occur by chance; i.e., axonal regeneration tends to be an all or none phenomenon. Furthermore, L-EGF boosted axonal regeneration of all three cell types while maintaining this coordination. This finding is not unanticipated in the case of VD2/VD3 and RPA neurons, considering the neurotrophic effect of L-EGF on these cells in vitro (Hermann et al. 2000b). It is unexpected, however, in the case of RPeD1, a cell type that does not respond with neurite outgrowth to the peptide in vitro. This result has two significant implications. First, it reiterates that one has to be careful in extrapolating in vitro data to the in vivo situation; i.e., a lacking neurotrophic response in vitro does not exclude in vivo activity of a growth factor. Second, it indicates that the effect of L-EGF on axonal regeneration may involve a non-neuronal intermediary that facilitates axonal regeneration in a global manner, regardless of the type of neuron. Alternatively, it may indicate that the response of individual neurons to L-EGF is conditioned by another factor(s) present in the brain.

The literature provides ample support for both of the above concepts. For example, EGF was reported to enhance outgrowth and survival of neonatal cerebellar neurons in vitro in the absence of glia (Morrison et al., 1988), whereas the neurotrophic actions of EGF on septal cholinergic neurons in culture depends on the presence of astrocytes (Kenigsberg and Mazzoni, 1995). Additionally, EGF supports the differentiation and proliferation of different glial populations, and the EGF/ErbB signaling system is involved in the activation of glia and regulation of glial-neuronal interactions after injury in mammalian and invertebrate nervous systems, a process thought to be required for axonal regeneration (Von Bernhardi and Muller, 1995; Yamada et al., 1997; Shafer et al., 1998; Streit et al., 1999; Xian and Zhou, 2000). The relevance of non-neuronal actions of L-EGF in axonal regeneration in the Lymnaea CNS remains to be determined, but is suggested by pilot studies indicating that L-EGF accelerates the accumulation of a microglia-like cell type in the area of the crush site (W. C. Wildering, unpublished observations).

Regarding conditional responses of neurons to growth factors, it is known that the neuronal responses to neurotrophins can depend on other factors such as cAMP levels or electrical activity (McAllister et al., 1996; Meyer-Franke et al., 1998). Moreover, evidence is mounting for extensive cross-talk between growth factor signaling pathways and extracellular matrix-associated signaling pathways; i.e., there are many options for ECM-dependent modulation of the response of a cell to growth factors (Plopper et al., 1995; Juliano, 1996; Wildering et al., 1997; Moro et al., 1998; Porter and Hogg, 1998). Obviously, with regards to the extracellular environment, the conditions prevailing in an in vitro culture system are substantially less complex than those normally experienced by neurons in situ. It is possible, therefore, that the intrinsic responsiveness of Lymnaea neurons to L-EGF is subject to modulation by other factors present in the organ-cultured CNS, an issue for investigation in future studies.

\section{REFERENCES}

Allison P, Benjamin PR (1985) Anatomical studies of central regeneration of an identified molluscan interneuron. Proc R Soc Lond B Biol Sci 226:135-157.

Ambron RT, Walters ET (1996) Priming events and retrograde injury signals. Mol Neurobiol 13:61-79.

Chalazonitis A, Kessler JA, Twardzik DR, Morrison RS (1992) Transforming growth factor $\alpha$, but not epidermal growth factor, promotes the survival of sensory neurons in vitro. J Neurosci 12:583-594.

Dubuisson AS, Beuermann RW, Kline DG (1993) Sciatic nerve regeneration across gaps within collagen chambers: the influence of epidermal growth factor. J Reconstr Microsurg 9:341-346.

Ebadi M, Bashir RM, Heidrick ML, Hamada FM, El Refaey H, Hamed A, Helal G, Baxi MD, Cerutis DR, Lassi NK (1997) Neurotrophins and their receptors in nerve injury and repair. Neurochem Int 30:347-374.

Fainzilber M, Smit AB, Syed NI, Wildering WC, Hermann PM, Van der Schors RC, Jiménez C, Li KW, Van Minnen J, Bulloch AGM, Ibáñez CF, Geraerts WPM (1996) CRNF, a molluscan neurotrophic factor that interacts with the p75 neurotrophin receptor. Science 274:1540-1543

Ferrair G, Toffano G, Skaper SD (1991) Epidermal growth factor exerts neuronotrophic effects on dopaminergic and GABAergic CNS neurons: comparison with basic fibroblast growth factor. J Neurosci Res 30:493-497.

Fredman SM (1987) Intracellular staining of neurones with nickel-lysine. J Neurosci Methods 20:181-194.

Frostick SP, Yin Q, Kemp GJ (1998) Schwann cells, neurotrophic factors, and peripheral nerve regeneration. Microsurgery 18:397-405.

Fry DW, Kraker AJ, McMichael A, Ambroso LA, Nelson JM, Leopold WR, Connors RW, Bridges AJ (1994) A specific inhibitor of the epidermal growth factor tyrosine kinase. Science 265:1093-1095.

Fu SY, Gordon T (1997) The cellular and molecular basis of peripheral nerve regeneration. Mol Neurobiol 14:67-116.

Goldberg JL, Barres BA (2000) The relationship between neuronal survival and regeneration. Annu Rev Neurosci 23:579-612.

Hermann PM, Wildering WC, Bulloch AGM (2000a) Functional recovery of respiratory behavior during axonal regeneration in snails ( $\mathrm{Lym}-$ naea stagnalis) is experience dependent. Behav Neurosci 114:410-423.

Hermann PM, van Kesteren RE, Wildering WC, Painter SD, Reno J, Smit JS, Kumar SB, Geraerts WPM, Ericsson LH, Smit AB, Bulloch AGM, Nagle GT (2000b) Neurotrophic actions of a novel molluscan epidermal growth factor. J Neurosci 20:6355-6364. 
Ide C (1996) Peripheral nerve regeneration. Neurosci Res 25:101-121.

Janse C, Kits KS, Lever AJ (1979) The re-formation of connections in the nervous system of Lymnaea stagnalis after nerve injury. Malacologia 18:485-488.

Janse C, Beek A, Van Oorschot I, Van der Roest M (1986) Recovery of damage in a molluscan nervous system is impaired with age. Mech Ageing Dev 35:179-183.

Juliano R (1996) Cooperation between soluble factors and integrinmediated cell anchorage in the control of cell growth and differentiation. BioEssays 18:911-917.

Justicia C, Planas AM (1999) Transforming growth factor-alpha acting at the epidermal growth factor receptor reduces infarct volume after permanent cerebral artery occlusion in rats. J Cereb Blood Flow Metab 19:128-132.

Kenigsberg RL, Mazzoni IE (1995) Identification of glial cell types involved in mediating epidermal growth factor's effects on septal cholinergic neurons. J Neurosci Res 41:734-744.

Kimpinski K, Mearow K (2001) Neurite growth promotion by nerve growth factor and insulin-like growth factor-1 in cultured adult sensory neurons: role of phosphoinositide 3-kinase and mitogen activated protein kinase. J Neurosci Res 63:486-499.

Kits KS, de Vries NJ, Ebberink RH (1990) Molluscan insulin-related neuropeptide promotes neurite outgrowth in dissociated neuronal cell cultures. Neurosci Lett 109:253-258.

Klaassen LJ, Janse C, van der Roest M (1998) Multiple synaptic connections of a single neuron change differentially with age. Neurobiol Aging 19:341-349.

Knüsel B, Hefti F (1992) K-252 compounds: modulators of neurotrophin signal transduction. J Neurochem 59:1987-1996.

Kruk PJ, Bulloch AGM (1992) Axonal regeneration of an identified Helisoma neuron depends on the site of axotomy. J Neurosci Res 3:401-412.

Logan A, Oliver JJ, Berry M (1994) Growth factors in CNS repair and regeneration. Prog Growth Factor Res 5:379-405.

McAllister AK, Katz LC, Lo DC (1996) Neurotrophin regulation of cortical dendritic growth requires activity. Neuron 17:1057-1064.

Meyer-Franke A, Wilkinson GA, Kruttgen A, Hu M, Munro E, Hanson Jr MG, Reichardt LF, Barres BA (1998) Depolarization and cAMP elevation rapidly recruit TrkB to the plasma membrane of CNS neurons. Neuron 21:681-693.

Moffett SB (1995) Neural regeneration in gastropod molluscs. Prog Neurobiol 46:289-330.

Morrison RS, Keating RF, Moskal JR (1988) Basic fibroblast growth factor and epidermal growth factor exert differential trophic effects on CNS neurons. J Neurosci Res 21:71-79.

Moro L, Venturino M, Bozzo C, Silengo L, Altruda F, Beguinot L, Tarone G, Defilippi P (1998) Integrins induce activation of EGF receptor: role in MAP kinase induction and adhesion-dependent cell survival. EMBO J 17:6622-6632.

Peng H, Wen TC, Tanaka J, Maeda N, Matsuda S, Desaki J, Sudo S,
Zhang B, Sakanaka M (1998) Epidermal growth factor protects neuronal cells in vivo and in vitro against transient forebrain ischemia- and free radical-induced injuries. J Cereb Blood Flow Metab 18:349-360.

Plopper GE, McNamee HP, Dike LE, Bojanowski K, Ingber DE (1995) Convergence of integrin and growth factor receptor signaling pathways within focal adhesion complex. Mol Biol Cell 6:1349-1365.

Porter JC, Hogg N (1998) Integrins take partners: cross-talk between integrins and other membrane receptors. Trends Cell Biol 8:390-396.

Raivich G, Bohatsek M, Kloss CUA, Werner A, Jones LL, Kreutzberg GW (1999) Neuroglial activation repertoire in the injured brain: graded response, molecular mechanisms and cues to physiological function. Brain Res Rev 30:77-105.

Rao G, Barnes CA, McNaughton BL (1986) Intracellular fluorescent staining with carboxyfluorescein: a rapid and reliable method for quantifying dye-coupling in mammalian central nervous system. J Neurosci Methods 16:251-263.

Riesse IIDJ, Stern DF (1998) Specificity within the EGF family/ErbB receptor family signaling network. BioEssays 20:41-48.

Rodgers W (1998) Analysis of cross-classified data. In: Reading and understanding multivariate statistics (Grimm LG, Yarnold PR, eds), pp 169-219. Washington, DC: American Psychological Association.

Ruegg UT, Burgess GM (1989) Staurosporine, K-252a and UCN-01: potent but non-specific inhibitors of protein kinases. Trends Pharmacol Sci 10:218-220

Shafer OT, Chen A, Kumar SM, Muller KJ, Sahley CL (1998) Injuryinduced expression of endothelial nitric oxide synthase by glial and microglial cells in the leech central nervous system within minutes after injury. Proc R Soc Lond B Biol Sci 265:2171-2175.

Streit WJ, Walter SA, Pennell NA (1999) Reactive microgliosis. Prog Neurobiol 57:563-581.

Terenghi G (1999) Peripheral nerve regeneration and neurotrophic factors. J Anat 194:1-14.

Toma JG, Pareek S, Barker P, Mathew TC, Murphy RA, Acheson A, Miller FD (1992) Spatiotemporal increases in epidermal growth factor receptors following peripheral nerve injury. $J$ Neurosci $12: 2504-2515$.

Von Bernhardi R, Muller KJ (1995) Repair of the central nervous system: lessons from lesions in leeches. J Neurobiol 27:353-366.

Wildering WC, Hermann PM, Bulloch AGM (1997) Rapid modulation of high-voltage-activated calcium currents by growth factors and RGDpeptides: a site of interaction between neurotrophic and extracellular matrix factors? Soc Neurosci Abstr 23:57.

Yamada M, Ikeuchi T, Hatanaka H (1997) The neurotrophic action and signaling of epidermal growth factor. Prog Neurobiol 51:19-37.

Xian CJ, Zhou X (1999) Neuronal-glial differential expression of TGFalpha and its receptor in the dorsal root ganglia in response to sciatic nerve lesion. Exp Neurol 157:317-326.

Xian CJ, Zhou X (2000) Role of transforming growth factor- $\alpha$ and related molecules in the nervous system. Mol Neurobiol 20:157-183. 